\title{
Survival of High Grade Glioma Patients Treated by Three Radiation Schedules with Chemotherapy: A Retrospective Comparative Study
}

\author{
Samir S. Mohamed ${ }^{1}$, Hamza A. Hamza ${ }^{2}$, Mohamed Alaa-Eldeen H. Mohamed ${ }^{1}$, Amany G. Ali' \\ 1 Clinical Oncology Department, Faculty of Medicine, Assiut University, Egypt; ${ }^{2}$ Radiotherapy \\ Department, South Egypt Cancer Institute, Faculty of Medicine, Assiut University, Egypt
}

Background: High grade glioma (HGG) is the most common primary malignant brain tumor. Radiotherapy (RT) plays an important role in the treatment of this tumor.

Aim: To compare the survival rates of HGG patients treated by conventional RT and those treated by hypofractionated or hyperfractionated RT combined with chemotherapy in two centers in Upper Egypt.

Methods: Data of HGG patients from two cancer care facilities in Upper Egypt who were treated by surgery followed by RT and temozolomide (TMZ) in the period between 2007 and 2012 were reviewed. Radiotherapy schedules were either conventional RT (60 Gy in 30 fractions over 6 weeks, group A) or hypofractionated RT (45 Gy in 15 fractions over 3 weeks, group B) or hyperfractionated RT ( $64.8 \mathrm{~Gy}$ in, $1.2 \mathrm{~Gy} /$ fraction, 2 fractions/day, group C) with \pm concurrent TMZ and adjuvant TMZ. Progression-free survival (PFS) and overall survival (OS) of patients after receiving the different types of RT treatment were evaluated.

Results: Forty-eight patients with grade III or IV HGG were identified. They were classified into 3 groups (A, B and C) that included 17, 16 and 15 patients, respectively. The median PFS were 6, 9 and 8 months $(p=0.354)$ and the median OS were 11,12 and $14(\mathrm{p}=0.760)$ for group A, B and C, respectively. Late RT toxicity was not different between the 3 groups. Conclusion: The three radiation schedules had a similar efficacy in adult HGG patients.

Keywords: Glioma, Chemoradiation, Radiation, External beam, Hypo-fractionation

Corresponding author: Mohamed Alaa-Eldeen H. Mohamed, MD; Clinical Oncology Department, Faculty of Medicine, Assiut University, Egypt; E-mail: malaaassiut@gmail.com

Submitted: 5-April-2017, Revised: 9-June-2017, Accepted: 11-June-2017

\section{INTRODUCTION}

High grade glioma (HGG) is the most common primary malignant brain tumor representing $40 \%$ of adult cases ${ }^{1}$. It affects more than 10,000 people in the United States and has an overall survival of 12-14 months ${ }^{2}$.

The current standard of care for HGG includes maximum safe excision followed by concurrent temozolomide (TMZ) and radiotherapy then adjuvant $\mathrm{TMZ}^{3}$

Radiation therapy (RT) after surgical resection increases the survival rates of patients with HGG compared with patients who were not irradiated ${ }^{4}$.

To avoid extensive neurologic toxicity, the standard safe radiation dose is considered 60 Gy with conventional fractionation schedule (1.8-2 Gy per fraction and 5 fractions per week) ${ }^{5}$.

Because of the short survival times of patients with glioblastoma, delivering the full therapeutic radiation dose within the shortest possible overall time is important. Subsequently, the accelerated hyperfractionated schedules are important for decreasing repopulation ${ }^{6}$. The limited life expectancy of patients with HGG requires evaluation of the hypo-fractionation schedules in order to shorten the time that patients spend receiving treatment for improving the quality of their life. ${ }^{7}$. Moreover, hypo-fractionation is associated with reduced costs compared to standard fractionation and has been accepted for elderly or poor performance patients ${ }^{6}$.

In this study, we aimed to investigate the clinical impact of three different RT schedules (conventional RT, hypofractionated RT and hyperfractionated RT) on the survival of adult HGG patients treated in two centers in Upper Egypt.

\section{METHODS}

This study was based on a retrospective analysis of the medical records of patients who underwent treatment for HGG at the Clinical Oncology Department, Faculty of Medicine, Assiut University and the Radiotherapy Department of South Egypt Cancer Institute, Assiut University in the period from January 2007 to December 2012.

The protocol of the study was reviewed and approved by the Ethics Committee of Assiut University before data collection.

\section{Inclusion criteria}

-Age at diagnosis between 16 and 70 years.

-Grade III or IV glial brain tumor according to the

World Health Organization (WHO) criteria ${ }^{8}$.

-Subtotal or partial resection was performed.

-The patients received postoperative chemoradiotherapy. 
-Available radio-chemotherapy data and follow-up data.

\section{Exclusion criteria}

-WHO grade I, II brain glioma

-Absence of histopathological data

-Patients underwent biopsy only

-Patients did not receive or complete radiochemotherapy schedule

-Absence of follow-up data

Data collected included age, sex, symptoms of disease (e.g., headache, epilepsy, upper and lower limb involvement), radiological examinations (magnetic resonance, multi-slice computed tomography and \pm magnetic resonance spectroscopy), site of the tumor, extent of surgery, histopathology, grade of tumor , radiotherapy fractionation, date of disease progression confirmed by radiology and the date of death or lost follow-up.

The outcomes of this study were progression-free survival (PFS) and overall survival (OS). PFS was calculated from the date of the start of radiochemotherapy to the date of the first documentation of disease progression. OS was calculated as the time period from diagnosis till death or the last follow-up visit.

Surgical intervention included either subtotal resection (over $75 \%$ of tumor resection) or partial resection (under $75 \%$ of tumor resection). The extent of resection based on the difference in preoperative and postoperative tumor volumes defined by radiology.

All patients received postoperative (6 weeks after surgery) radio-chemotherapy. The chemotherapy treatment plan included TMZ.

Patients in the hypo-fractionation arm were those from far locality preferring shorter treatment period, in addition to those with poor performance status.

After simulation and immobilization using the thermoplastic mask, external beam radiation therapy (teletherapy) was delivered either by Cobalt-60 or linear accelerator machine (6 MV).

The irradiated area covered the tumor bed, residual enhancing tumor as seen on post-surgical magnetic resonance imaging scan and surrounding edema plus a margin of $2 \mathrm{~cm}$. All patients were treated with either parallel-opposing or oblique-wedged fields. Coning down for targeted volume to cover residual enhancing tumor plus a safety margin of $2 \mathrm{~cm}$ was used in the conventional arm after $41.4 \mathrm{~Gy}$.

Radiation therapy was given concomitantly with TMZ at a dose of $75 \mathrm{mg} / \mathrm{m} 2$ daily in cases with grade IV glioma and after RT for all cases. Temozolomide was given after radiotherapy with a dose of $150 \mathrm{mg} / \mathrm{m} 2$ daily for 5 days every 28 days for 6 cycles.

\section{Toxicity assessment}

Assessment of toxicity of chemotherapy (TMZ) was done according to National Cancer Institute Common Toxicity Criteria version- ${ }^{9}$. Late radiation toxicity was assessed according to the radiation morbidity scoring schema of Radiation Therapy Oncology Group ${ }^{10}$.

Patients were divided into three groups (A, B and C). Members group A were treated by conventional RT (60 Gy, 1.8-2 Gy/ fraction daily, 5 days per week, over 6-7 weeks,). Patients in group B were treated by hypofractionated RT (45 Gy, 3 Gy /fraction, 5 days per week, over 3 weeks). In group C, patients were treated by hyperfractionated radiotherapy $(64.8 \mathrm{~Gy}, 1.2 \mathrm{~Gy}$ /fraction, 2 fractions per day with an interval of 6-8 hours, 5 days per week).

\section{Statistical analysis}

Data expressed as numbers, percentages and medians. Clinical characteristics were compared between the three groups by Chi-square test. PFS and OS were estimated by Kaplan-Meier method and compared by Log-rank test. Statistically significant Pvalues were considered less than 0.05. Statistical analysis was performed by using SPSS version 20 (SPSS, Inc., Chicago. IL).

\section{RESULTS}

Forty-eight patients were eligible for the study. The conventional radiotherapy (group A) included 17 patients versus 16 patients included in the hypofractionated schedule (group B) and 15 in the hyperfractionated schedule (group C).

Characteristics of the 48 patients are shown in table 1. The mean age of all patients was 46.4 years $( \pm$ 13.6).The presenting symptom was headache in 31 (64.6\%) patients, convulsions in 12 (25\%), upper limb paresis in $17(35.4 \%)$ and lower limb paresis in 15 $(31.3 \%)$.

The three groups of patients were balances in terms of patient and tumor characteristics.

Table 2 shows the median and 95\% confidence interval of OS and PFS of patients in the three treatment groups. No statistical significance differences revealed between the three groups of patients that were treated with 3 different fractionated schedules of radiotherapy. A Kaplan-Meier estimate of PFS of the study cohort of patients with HGG according to radiation schedules is presented in figure 1. Overall survival curves of the study cohort according to the three fractionation schedules of RT are shown in figure 2.

Table 3 demonstrates the survival and the status of HGG among our patients at 12 months.

Grade II hematological toxicity of chemotherapy developed in $4(23.5 \%), 5(31.3 \%)$ and $3(20 \%)$ patients in groups A, B and C, respectively. Grade II alimentary adverse events recorded in $3(17.6 \%), 2(12.5 \%)$ and 3 (20\%) patients in the 3 groups respectively. Grade III radiation toxicity occurred in $3 / 16$ patients treated with hypofractionated RT, 2/15 patients in hyperfractionated $\mathrm{RT}$ arm and none in conventional RT. 
Table 1: Characteristics of patients

\begin{tabular}{|c|c|c|c|c|}
\hline & Total & $\begin{array}{l}\text { Group A } \\
\text { (Conventional RT) }\end{array}$ & $\begin{array}{l}\text { Group B } \\
\text { (Hypofractionated RT ) }\end{array}$ & $\begin{array}{l}\text { Group C } \\
\text { (Hyperfractionated RT) }\end{array}$ \\
\hline & No. (\%) & No. $(\%)$ & No. $(\%)$ & No. $(\%)$ \\
\hline Number & $48(100)$ & $17(35.4)$ & $16(33.3)$ & $15(31.3)$ \\
\hline \multicolumn{5}{|l|}{ Age } \\
\hline$\leq \mathbf{5 0}$ & $30(62.5)$ & $14(82.4)$ & $7(43.8)$ & $9(60)$ \\
\hline$>50$ & $18(37.5)$ & $3(17.6)$ & $9(56.2)$ & $6(40)$ \\
\hline \multicolumn{5}{|l|}{ Sex } \\
\hline Male & $34(70.8)$ & $13(76.5)$ & $13(81.3)$ & $8(53.3)$ \\
\hline Female & $14(29.2)$ & $4(23.5)$ & $3(18.7)$ & $7(46.7)$ \\
\hline \multicolumn{5}{|l|}{ Anatomical site } \\
\hline Parietal & $37(54.4)$ & $10(14.7)$ & $12(17.6)$ & $15(22.1)$ \\
\hline Frontal & $13(19.1)$ & $5(7.4)$ & $5(7.4)$ & $3(4.4)$ \\
\hline Temporal & $10(14.7)$ & $4(5.9)$ & $3(4.4)$ & $3(4.4)$ \\
\hline Other & $8(11.8)$ & $3(4.4)$ & $4(5.9)$ & $1(1.5)$ \\
\hline \multicolumn{5}{|l|}{ Tumor grade } \\
\hline III & $14(29.2)$ & $5(29.4)$ & $5(31.3)$ & $4(26.7)$ \\
\hline IV & $34(70.8)$ & $12(70.6)$ & $11(68.7)$ & $11(73.3)$ \\
\hline \multicolumn{5}{|l|}{ Surgery } \\
\hline Subtotal resection & $44(91.7)$ & $16(94.1)$ & $14(87.5)$ & $14(93.3)$ \\
\hline Partial excision & $4(8.3)$ & $1(5.9)$ & $2(12.5)$ & $1(6.7)$ \\
\hline
\end{tabular}

Table 2: Survival rates of the three fractionation schedules of radiotherapy

\begin{tabular}{|c|c|c|c|c|}
\hline & $\begin{array}{l}\text { Group A } \\
\text { (Conventional RT) }\end{array}$ & $\begin{array}{l}\text { Group B } \\
\text { (Hypofractionated RT) }\end{array}$ & $\begin{array}{l}\text { Group C } \\
\text { (Hyperfractionated RT) }\end{array}$ & P-value \\
\hline & \multicolumn{3}{|c|}{ Median number of months (95\% Confidence interval) } & \\
\hline Progression free survival & $6(4.38-7.61)$ & $9(8.09-9.9)$ & $8(6.48-9.51)$ & 0.573 \\
\hline Overall survival & $11(9.67-12.32)$ & $12(8.08-15.92)$ & $14(3.22-7.68)$ & 0.76 \\
\hline
\end{tabular}

Table 3: Survival and disease status of high grade glioma patients at 12 months according to fractionation schedule

\begin{tabular}{|c|c|c|c|c|}
\hline & $\begin{array}{l}\text { Group A } \\
\text { (Conventional RT) }\end{array}$ & $\begin{array}{l}\text { Group B } \\
\text { (Hypofractionated RT) }\end{array}$ & $\begin{array}{l}\text { Group C } \\
\text { (Hyperfractionated RT) }\end{array}$ & P-value \\
\hline & No. $(\%)$ & No. $(\%)$ & No. $(\%)$ & \\
\hline Died & $10(58.8)$ & $6(37.5)$ & $6(40)$ & \multirow[t]{3}{*}{0.6} \\
\hline Alive with progressive disease & $3(17.7)$ & $5(31.3)$ & $3(20)$ & \\
\hline Alive with stable disease & $4(23.5)$ & $5(31.3)$ & $6(40)$ & \\
\hline
\end{tabular}

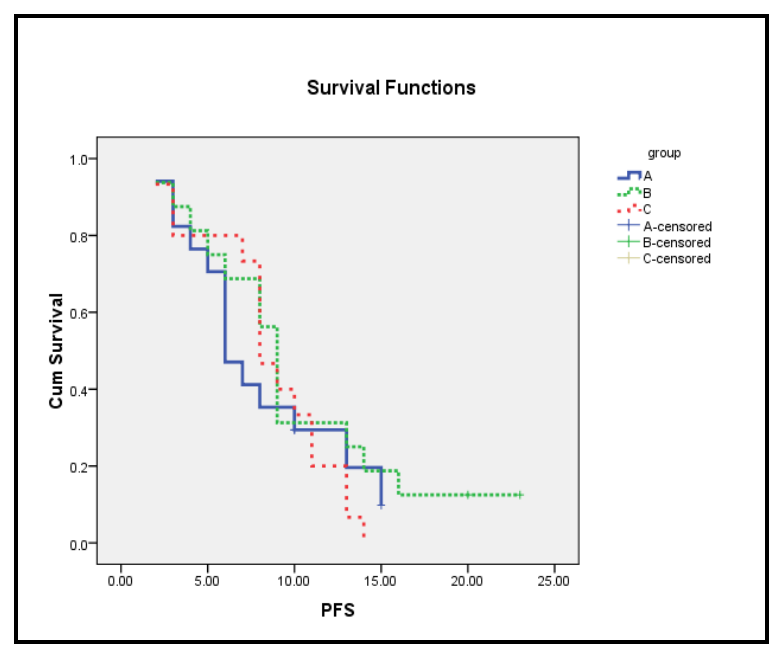

Figure 1: Progression-free survival (PFS) in months by treatment group (group A: conventional RT, group B: Hypofractionated RT \& group C: Hyperfractionated)

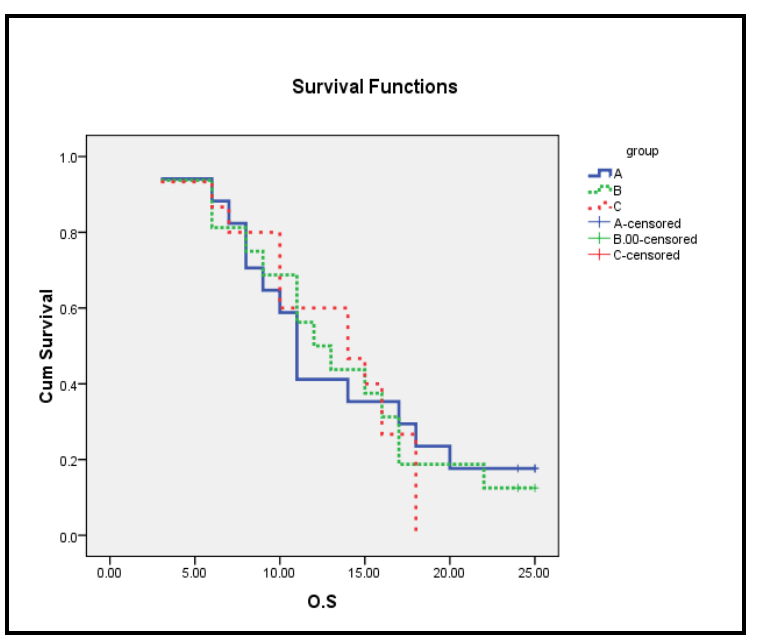

Figure 2: Overall survival (OS) in months by treatment group (group A: conventional RT, group B: Hypofractionated RT \& group C: Hyperfractionated) 


\section{DISCUSSION}

Dose escalation by hyperfractionated or accelerated hyperfractionated RT has been tested in HGG. Some studies recommended using the hypofractionated RT instead of conventional and hyperfractionated radiotherapy. Although altered fractionation RT schedules may shorten the overall treatment-time of HGG patients, there is no significant survival improvement ${ }^{11}$.

In our retrospective study, we evaluated the survival rates of adult patients diagnosed with HGGs and treated with different fractionation schedules of radiotherapy followed by TMZ in two centers in Upper Egypt.

Regarding the incidence of HGGs according to age, $62.5 \%$ of our patients were $\leq 50$ years and $37.5 \%$ were $>50$ years old. These results were comparable to the results by Chen et al 201512 whereas patients $\leq 50$ years and $>50$ years old constituted $52.8 \%$ and $47.2 \%$, respectively. The study of Chen et al ${ }^{12}$ showed that regardless of the definition of age groups, older patients has a significantly lower survival rates.

With respect to sex, in our current work the distribution of HGGs in females was $29.2 \%$ versus $70.8 \%$ in males. Alike, Chen et al ${ }^{12}$ reported a higher percentage of males diagnosed with HGGs than females $(62.4 \%$ in males, $37.6 \%$ in females) and the survival outcomes were better in females.

Regarding the anatomical sites of HGGs, parietal region was the commonest site among our patients as represented in $54.4 \%$ of cases followed by frontal region $(19.1 \%)$ and temporal region $(14.7 \%)$. On the contrary, Nomiya et al ${ }^{13}$ reported the frontal site as the most common site $(52 \%)$ followed by temporal $(21 \%)$ and parietal $(7 \%)$.

In our study, there was no significant difference between hyperfractionated RT and conventional RT regarding PFS and OS. The median PFS was 8 months for the hyperfractionated RT versus 6 months for conventional RT with median OS 14 months and 11 months, respectively. Buckner et al ${ }^{14}$ found that accelerated RT with $1.60 \mathrm{~Gy}$ twice daily for 2 weeks was non-inferior to standard RT of 1.80 Gy daily for 5 weeks with comparable safety and efficacy.

We found no significant difference between hyperfractionated RT and conventional RT regarding radiation induced toxicity. Only $2 / 16$ patients treated with hyperfractionated RT presented with grade III toxicity. This is consistent with Shibamoto et al ${ }^{15}$ who observed brain necrosis in $40 \%$ (4/10) of patients treated with hyperfractionated RT schedule (69 Gy, 1.5 Gy per fraction twice daily); while none of those treated with conventional fractionation (64.8 Gy, 1.8 Gy per fraction) had brain necrosis.

In our study, 16 patients with HGG were treated with a hypofractionated RT regimen obtaining a median OS and PFS of 12 and 9 months, respectively. No significant difference revealed from patients treated with conventional RT, who showed a median OS and PFS of
11 and 6 months. Three of our patients developed grade III late radiation toxicity after hypofractionated RT. Our results were comparable to that obtained by Malmstrom et al $^{7}$ who showed no difference in elderly patients $(\geq 60$ years) between hypo-fractionated RT (34 Gy, 3.4 Gy per fraction, over 2 weeks) compared with standard fractionation (60 Gy, 2.0 Gy per fraction).

The comparable survival results of hypofractionated RT and conventional RT was also reported by Arvold et al ${ }^{16}$. Besides, asymptomatic radiation necrosis was reported in 4/12 patients 9-31 months post-irradiation after hypofractionated RT ${ }^{17}$. Conversely, Sultanem et al 18 studied a series of 25 patients treated with intensitymodulated radiotherapy in a hypofractionated protocol. Treatments were well tolerated, and no acute or late toxicities were observed during follow-up.

\section{Conclusion}

This retrospective study suggests a non-significant difference in survival rates between hyperfractionated and hypofractionated radiotherapy when compared with conventional radiotherapy with chemotherapy in the treatment of HGG in adults.

Further research is needed that should include other treatment outcomes like quality of life and overall toxicity.

\section{REFERENCES}

1. Panet-Raymond V, Souhami L, Roberge D, et al. Accelerated hypofractionated intensity-modulated radiotherapy with concurrent and adjuvant temozolomide for patients with glioblastoma multiforme: a safety and efficacy analysis. Int J Rad Oncol Biol Physics. 2009; 73(2):473-478.

2. Dolecek TA, Propp JM, Stroup NE, Kruchko C. CBTRUS statistical report: primary brain and central nervous system tumors diagnosed in the United States in 2005 2009. Neuro-Oncol. 2012; 14(Suppl 5): v1-v49.

3. Stupp R, Mason WP, van den Bent MJ, et al. European Organisation for Research and Treatment of Cancer Brain Tumor and Radiotherapy Groups; National Cancer Institute of Canada Clinical Trials Group. Radiotherapy plus concomitant and adjuvant temozolomide for glioblastoma. N Engl J Med. 2005; 352(10): 987-996.

4. Sheline GE. Radiation therapy of brain tumors. Cancer. 1977; 39(2 Suppl): 873-881.

5. Dhormain F. Radiotherapy of high-grade gliomas: current standards and new concepts, innovations in imaging and radiotherapy, and new therapeutic approaches. Chin J Cancer. 2014; 33(1): 16-24.

6. Roa W, Xing JZ, Small C, et al. Current developments in the radiotherapy approach to elderly and frail patients with glioblastoma multiforme. Expert Rev Anticancer Ther. 2009; 9(11): 1643-1650.

7. Malmstrom A, Gronberg $\mathrm{BH}$, Marosi $\mathrm{C}$, et al. Temozolomide versus standard week radiotherapy versus hypofractionated radiotherapy in patients older than 60 years with glioblastoma: the Nordic randomised, phase 3 trials. Lancet Oncol. 2012; 13(9):916-926.

8. Louis DN, Ohgaki H, Wiestler OD, et al. The 2007 WHO classification of tumors of the central nervous system, Acta Neuropathol. 2007; 114(2): 97-109. 
9. Common Toxicity Criteria classification v3.0. Available from:

https://ctep.cancer.gov/protocoldevelopment/electronic_a pplications/docs/ctcaev3.pdf

10. Cox J, Stez J. Toxicity criteria of the Radiation Therapy Oncology Group (RTOG) and the European organization for research and treatment of cancer (EORTC). Int J Rad Oncol Biol Phys. 1995; 31(5):1341-1346.

11. Nieder C, Andratschke N, Wiedenmann $\mathrm{N}$, et al. Radiotherapy for high-grade gliomas. Does altered fractionation improve the outcome? Strahlenther Onkol. 2004; 180(7): 401-407.

12. Chen JW, Zhou CF, Lin ZX. The influence of different classification standards of age prognosis in high-grade hemispheric glioma patients. J Neurol Sci. 2015; 356(12): $148-152$

13. Nomiya $T$, Nemoto $K$, Kumabe $T$, et al. Prospective single-arm study of 72 Gy hyperfractionated radiation therapy and combination chemotherapy for anaplastic astrocytomas. BMC Cancer. 2008; 8: 11.

14. Buckner JC, Ballman KV, Michalak JC, et al. Phase III trial of carmustine and cisplatin compared with carmustine alone and standard radiation therapy or accelerated radiation therapy in patients with glioblastoma multiforme: North Central Cancer Treatment Group 9372-52 and Southwest Oncology Group 9503 Trials. J Clin Oncol. 2006; 24(24): 3871-3879.

15. Shibamoto Y, Nishimura Y, Tsutsui K, et al. Comparison of accelerated hyperfractionated radiotherapy and conventional radiotherapy for supratentorial malignant glioma. Jpn J Clin Oncol. 1997; 27(1):31-36.

16. Arvold ND, Tanguturi SK, Aizer AA, et al. Hypofractionated versus standard radiation therapy with or without temozolomide for older glioblastoma patients. Int J Radiat Oncol Biol Phys. 2015; 92(2): 384-389.

17. Cha J, Suh CO, Park K, et al. Feasibility and Outcomes of Hypofractionated Simultaneous Integrated Boost-Intensity Modulated Radiotherapy for Malignant Gliomas: A Preliminary Report. Yonsei Med J. 2014; 55(1): 70-77.

18. Sultanem K, Patrocinio H, Lambert C, et al. The use of hypofractionated intensity-modulated irradiation in the treatment of glioblastoma multiforme: preliminary results of a prospective trial. Int J Radiat Oncol Biol Phys. 2004; 58(1): 247-252. 